\title{
Mesure de la masse volumique de l'eau interfoliaire dans les smectites; application aux courbes capillaires
}

Commissariat à l'énergie atomique, Centre d'études de Cadarache DTN/SMTM/LMTE

Bâtiment 307 13108 Saint-Paul-lez-Durance joel.raynal@cea.fr

\begin{abstract}
Dans cette étude, le comportement du système argile-eau est abordé à partir des potentiels hydriques correspondant au domaine des humidités relatives comprises entre 5 et $97 \% \mathrm{HR}$, soit un potentiel de succion entre 400 et $4 \mathrm{MPa}$, en utilisant cinq smectites sous forme de poudre. Dans ces conditions, l'espace interagrégats n'est pas saturé par de l'eau. Par contre, la variation de la distance basale $d_{001}$ des feuillets argileux est concommitante avec une évolution de la teneur en eau du matériau, montrant que c'est l'espace interfoliaire qui est le principal siège de l'hydratation. A travers la détermination de la masse volumique de solide, à l'aide d'un pycnomètre à hélium, la masse volumique de cette eau interfoliaire a pu être déterminée expérimentalement. Quand on détermine le volume total d'une poudre en pycnométrie sur un matériau argileux, il est nécessaire d'effectuer une correction pour tenir compte du volume occupé par l'eau. Les premières corrections, utilisant une masse volumique de l'eau égale à celle de l'eau pure, n'ont pas donné de résultats satisfaisants. Pour retrouver les données expérimentales, il est nécessaire de prendre en compte une masse volumique de l'eau interfoliaire comprise entre 1,14 et $1,18 \mathrm{mg} / \mathrm{m}^{3}$. Cette valeur dépend notamment de la composition de l'ion échangeable et, en particulier, du rapport sodium/calcium échangeables. Une telle valeur de masse volumique de l'eau aurait en particulier pour conséquence une diminution de 10-15\% du degré de saturation et une modification des courbes capillaires.
\end{abstract}

Mots clés: smectite, masse volumique, eau, eau interfoliaire, succion, argiles.

The purpose of this work is to study the particular physical properties of interlayer water in clay-water systems. Five smectites, in a powder form, are studied at different hydric potentials in a range of relative humidity between 5 and $97 \%$. These relative humidities correspond to a suction potential between $400 \mathrm{MPa}$ and $4 \mathrm{MPa}$.

Regardless of these RH conditions, the interaggregates space is never saturated with water. We also observed that the basal spacing $d_{001}$ of the clay sheets changes in accordance with the evolution of water content. These facts lead us conclude that 
the interlayer space is the main location where the hydratation occurs, i.e. where we expect the physical properties such as the water density to be different from bulk water.

The density of the interlayer water and the solid density are simultaneously measured by means of a helium pycnometer. When measuring the total volume of a powder with this method, a correction is needed to take into account the volume occupied by water. Corrections using a density of the water equal to that of pure water did not give satisfactory results. To attain an agreement with the experimental results, we had to recalculate the density of the interlayer water. These values are found to lie between 1,14 and $1,18 \mathrm{Mg} /{ }^{\beta}$ for the studied clays and depend on the population of exchangeable cations present in the interlayer space, especially on the sodium/calcium ratio. The consequences of a such water density upon suction curves (as a decrease of $10-15 \%$ on the saturation degree) are discussed.

Key words : smectite, density, water, interlayer water, suction, clays.

1

\section{Introduction}

Dans les concepts classiques de mécanique des sols appliqués aux matériaux argileux gonflants, la courbe de succion capillaire fait appel aux phénomènes de capillarité entre deux particules argileuses, ou entre deux agrégats argileux. Or, Tessier (1984) souligne que la notion de ( particule argileuse » peut prendre divers aspects non seulement en fonction du potentiel hydrique (potentiel de succion), mais aussi en fonction de la nature du matériau argileux et du cation échangeable. Les matériaux argileux gonflants et, plus particulièrement, les smectites possèdent une teneur en eau qui varie très fortement en fonctions des conditions hygrométriques. En dessous de $5 \%$ HR (et donc pour les très fortes succions : $>400 \mathrm{MPa}$ ), les smectites sont faiblement hydratées et se réhydratent rapidement dès qu'elles sont remises en contact avec l'humidité ambiante. Dans le domaine des fortes humidités relatives (au-dessus de 95-97 \% HR), la teneur en eau évolue très vite pour un faible incrément de l'humidité relative. La courbe de sorption d'eau $w=\mathrm{f}(\% \mathrm{HR})$ y est très ( raide », notamment pour les smectites sodiques. Dans ce domaine, qui est obtenu en utilisant des techniques pères (plaques tensiométriques, dispositifs de filtration, presse à membrane (Ben Rhaiem et al., 1986; Tessier, 1984), voire directement dans l'eau), les vides existants sont pratiquement tous remplis par l'eau : le degré de saturation est proche de 1.

Dans cette étude, seul le domaine des humidités relatives utilisant des solutions salines saturées est exploré. A priori, le gonflement des matériaux argileux dans ce domaine est faible par rapport à celui des fortes HR (proches de $100 \%$ ). Cependant, l'évolution de la teneur en eau dans ce domaine n'est pas négligeable. De plus, les études en diffraction de rayons X (DRX) montrent que la distance basale $d_{o o t}$ varie également entre 5 et $95 \%$ HR (Glaeser et Mering, 1968 ; Moore et Reynolds, 1997) : les variations de teneur en eau sont donc à relier avec l'eau présente dans l'espace interfoliaire, et non avec l'eau interagrégats ou interparticulaire. Cette eau interfoliaire est liée par des liaisons Van der Walls aux cations interfoliaires qui compensent le déficit de charge du feuillet de la smectite. Elle est davantage liée au ( matériau solide » argileux (le feuillet) que l'eau interparticulaire. Cependant, il est pratiquement impossible de distinguer par des moyens usuels ces différents types d'eau. En particulier, la détermination de la teneur en eau, telle qu'elle est classiquement effectuée par perte au feu à 105 ou $110^{\circ} \mathrm{C}$, est celle de la quantité d'eau globale, sans distinction entre l'eau interfoliaire et les eaux interparticulaires et interagrégats, alors qu'il est intuitif qu'il s'agit d'états différents. Par exemple, Ben Rhaiem et al. (1986) établissent pour une argile du Wyoming calcique portée à une contrainte hydrique de 0,32 $\mathrm{MPa}(99,9 \%$ d'humidité relative) la répartition suivante : $84,5 \%$ d'eau externe (située dans les pores interparticulaires) et $15,5 \%$ d'eau interne, elle-même subdivisée en eau contenue dans les espaces interfoliaires $(13 \%)$ et dans les pores lenticulaires $(2,5 \%)$.

D'autres auteurs (Berend, 1991 ; Robinet et al., 1997) utilisent soit les mêmes termes (eau interne/eau externe), soit les concepts (c eau libre »/( ( eau liée ») pour établir une distinction entre les différents types d'eau, ce qui pourrait être expliqué par le fait qu'ils n'emploient pas les mêmes méthodes et proposent des définitions différentes de l'eau. L'intérêt scientifique de ces études est de pouvoir caractériser le système argile-eau, dans le but de modéliser les phénomènes de rétention-transport. Les différents modèles essaient de prendre en compte la structure particulière de ce système (aux différentes échelles) pour prédire la migration d'éléments chimiques dans un milieu argileux (par exemple dans le cas d'un stockage de déchets radioactifs ou dans la description du transfert des polluants dans les sols).

L'objet de cette étude n'est pas d'établir clairement une distinction entre ces différents types d'eau, mais juste de mesurer une propriété physique (la masse volumique de l'eau interfoliaire) et démontrer ainsi expérimentalement qu'elle se distingue de l'eau « normale ». Ceci est réalisé en mesurant avec un pycnomètre à hélium la masse volumique de solide (qui est un paramètre nécessaire pour calculer en mécanique des sols des paramètres majeurs tels que la porosité, l'indice des vides ou le degré de saturation). In fine, les conséquences sur les lois de comportement hydromécanique des argiles gonflantes seront évaluées.

\section{2}

\section{Matériaux}

Cinq matériaux pulvérulents à base de smectite sont étudiés. Il s'agit de deux montmorillonites sodiques du 
Wyoming (WYO1 et WYO2) ; deux montmorillonites calciques grecque (GREC) et turque (TURK) et une beidellite calcique de Provence (PROV). Aucune purification ni saturation mono-ionique n'a été effectuée : tous les essais ont été effectués sur les matériaux bruts. Les principales phases identifiées par diffraction de rayons X (diffractomètre Siemens D500 avec tube cobalt) montrent la présence d'impuretés non argileuses, à l'exception de l'échantillon TURK qui présente une pureté exceptionnelle pour un matériau smectitique ( en vrac ») (Tableau I). La distinction entre une beidellite (smectite gonflante avec déficit de charge localisé en couche tétraédrique) et une montmorillonite (smectite gonflante avec déficit de charge localisé en couche octaédrique) est réalisée par analyse thermique (Chantret et al., 1971; Trauth, 1964). Les courbes d'analyse thermique effectuée avec une thermobalance simultanée Netzsch STA409 couplée à un spectromètre de masse quadripolaire Balzers Thermostar 300 (Raynal, 1989) et les spectres de diffraction X sont en cours de publication. Les valeurs de capacité d'échange cationique, mesurée par la méthode à la cobalthexaammine, à l'INRA, confirment la richesse en smectite de l'échantillon TURK, qui est pratiquement celui d'un matériau pur (Tableau II).

\begin{tabular}{c|l}
\multicolumn{2}{c}{$\begin{array}{l}\text { TABLEAUI } \\
\text { Caractéristiques minéralogiques des cinq } \\
\text { smectites étudiées. }\end{array}$} \\
Échantillon & Principaux composés (DRX + analyse thermique) \\
\hline GREC & $\begin{array}{l}\text { Montmorillonite Ca ; cristobalite ; feldspaths ; } \\
\varepsilon \text { quartz, } \varepsilon \text { calcite }\end{array}$ \\
\hline PROV & Beidellite $\mathrm{Ca}$; quartz ; $\varepsilon$ calcite \\
\hline TURK & Montmorillonite $\mathrm{Ca}$ \\
\hline WYO1 & $\begin{array}{l}\text { Montmorillonite } \mathrm{Na} \text {; quartz; feldspaths ; } \\
\varepsilon \text { gypse ; cristobalite? }\end{array}$ \\
\hline WYO2 & Montmorillonite $\mathrm{Na}$; quartz; feldspaths
\end{tabular}

\section{3}

\section{Principe de mesure}

La mesure du volume est effectuée par un pycnomètre à déplacement de fluide. Le fluide est un gaz qui pénètre dans les pores les plus petits, de l'ordre de l'angström. L'hélium est généralement utilisé, car, étant un gaz inerte, il présente également l'avantage de ne pas être sujet à des interactions physico-chimiques avec les solides.
Le schéma de principe d'un pycnomètre à gaz est présenté à la figure 1 où $V_{\text {cell }}$ est le volume de la cellule contenant l'échantillon de volume $\mathrm{V}$, et $\mathrm{V}_{\text {add }}$ un volume additionnel, à la pression atmosphérique $\mathrm{P}_{\text {atm }}$ et à la température ambiante $\mathrm{T}_{\mathrm{amb}}$.

Dans un premier temps (Fig. 1), on ferme la vanne de communication $\mathrm{v}_{\mathrm{c}}$ et on augmente la pression dans la cellule par injection d'hélium (vanne $v_{\text {inj }}$ ouverte) jusqu'à une valeur $\mathrm{P}_{1}$ (préprogrammée à environ $0,15 \mathrm{MPa}$ relatifs), à laquelle $\mathrm{v}_{\text {inj }}$ est fermée. Les conditions dans la cellule sont telles que la loi des gaz parfaits est respectée, le nombre de moles d'hélium $n_{H e}$ dans la cellule est donc à l'équilibre (zone gris clair de la figure 1):

$$
P_{1} \cdot\left(V_{\text {cell }}-V\right)=n_{H e} \cdot R \cdot T_{a m b}
$$
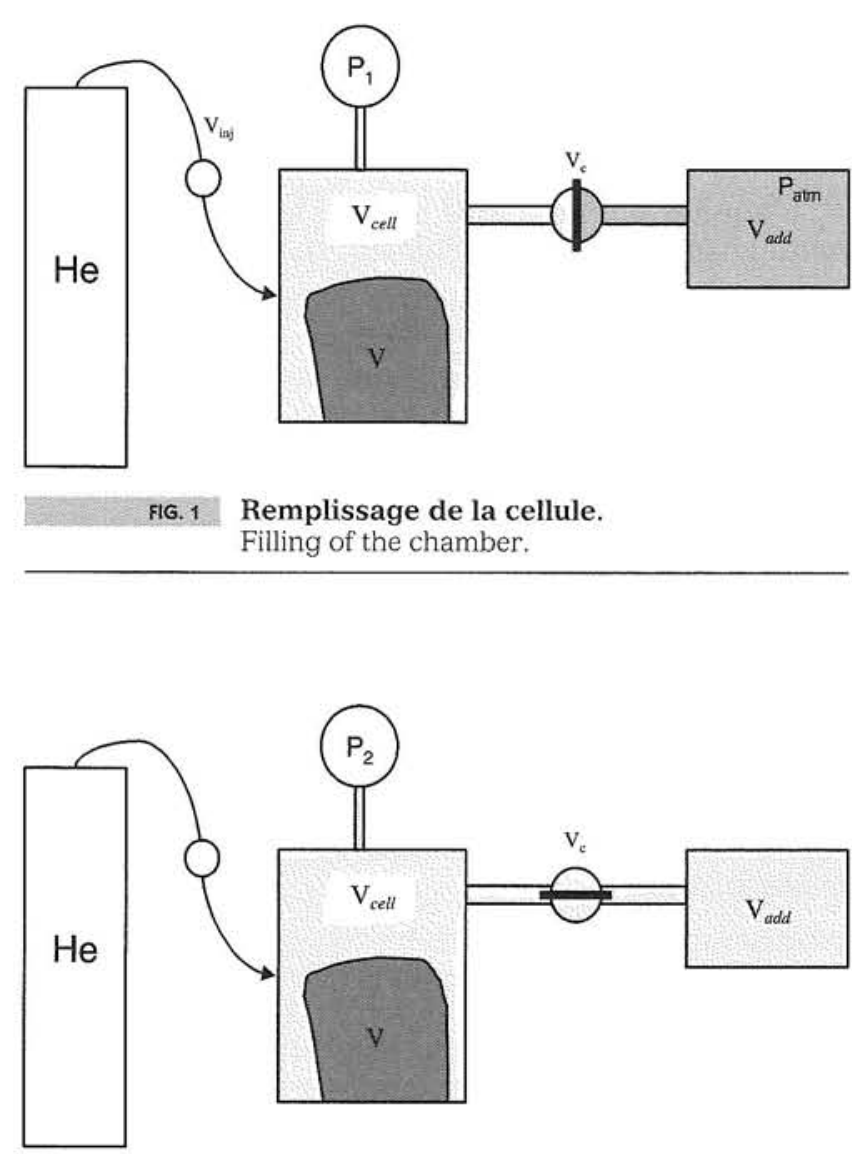

FG. 2 Répartition de l'hélium dans le volume additionnel.

Helium repartition in the expansion volume.

TABLEAUII Capacité d'échange et ions échangeables des cinq smectites (en ceq/kg).

\begin{tabular}{c|c|c|c|c|c} 
Échantillon & CEC & $\mathrm{Ca}$ & $\mathrm{Mg}$ & $\mathrm{K}$ & $\mathrm{Na}$ \\
\hline GREC & 68,4 & 29,0 & 9,7 & 2,8 & 23,3 \\
\hline PROV & 67,0 & 61,8 & 5,3 & 1,5 & 0,1 \\
\hline TURK & 117,4 & 77,5 & 12,1 & 1,3 & 26,6 \\
\hline WYO1 & 84,8 & 25,6 & 5,2 & 1,5 & 58,9 \\
\hline WYO2 & 83,9 & 21,0 & 4,2 & 21,0 & 51,6
\end{tabular}


Le volume additionnel étant resté à la pression atmosphérique, l'équation d'état s'y appliquant est ( $n_{\text {add }}$ étant le nombre de moles de gaz dans $V_{\text {add }}$ ):

$$
P_{\text {atm }} \cdot V_{\text {add }}=n_{\text {add }} \cdot R \cdot T_{\text {amb }}
$$

Dans un deuxième temps, la vanne de communication $v_{c}$ est ouverte (Fig. 2) : les $n_{H e}$ moles d'hélium se répartissent dans la cellule de mesure et dans le volume additionnel $\mathrm{V}_{\text {add }}$, entraînant une baisse de la pression mesurée, qui se stabilise à une valeur $\mathrm{P}_{2}$. L'équation du système est alors :

$$
P_{2} \cdot\left(V_{\text {cell }}-V+V_{\text {add }}\right)=n_{H e} \cdot R \cdot T_{\text {amb }}+n_{\text {add }} \cdot R \cdot T_{\text {amb }}
$$

En substituant les équations 1 et 2 dans l'équation 3 , on a:

$$
P_{2} \cdot\left(V_{\text {cell }}-V+V_{\text {add }}\right)=P_{1} \cdot\left(V_{\text {cell }}-V\right)+P_{\text {atm }} \cdot V_{\text {add }}
$$
ou

$$
\left(P_{2}-P_{1}\right) \cdot\left(V_{\text {cell }}-V\right)=\left(P_{\text {atm }}-P_{2}\right) \cdot V_{\text {add }}
$$

puis

$$
V_{\text {cell }}-V=\frac{\left(P_{\text {atm }}-P_{2}\right)}{P_{2}-P_{1}} \cdot V_{\text {add }}
$$

L'équation 6 peut être réduite en ajoutant et en retranchant $\mathrm{P}_{\text {atm }}$ au dénominateur, ce qui donne :

$$
V_{\text {cell }}-V=\frac{\left(P_{\text {atm }}-P_{2}\right) \cdot V_{\text {add }}}{\left(P_{2}-P_{\text {atm }}\right)-\left(P_{1}-P_{\text {atm }}\right)}
$$

En divisant par $\left(\mathrm{P}_{\text {atm }}-\mathrm{P}_{2}\right)$ au numérateur et au dénominateur, on a :

$$
V-V_{\text {cell }}=\frac{V_{\text {add }}}{\frac{P_{1}-P_{\text {atm }}}{P_{2}-P_{\text {atm }}}-1}
$$

$\mathrm{P}_{1}, \mathrm{P}_{2}$ et $\mathrm{P}_{\mathrm{atm}}$ sont exprimées en pressions absolues. Cependant, le capteur utilisé est un capteur de pression relative, pour lequel $\mathrm{P}_{\mathrm{atm}}$ est réglé à zéro ; les pressions lues sont alors $\mathrm{P}_{1 \mathrm{~g}}$ et $\mathrm{P}_{2 \mathrm{~g}}$. L'équation 8 se simplifie donc finalement en :

$$
V-V_{\text {cell }}+\frac{V_{a d d}}{1-\frac{P_{1 g}}{P_{2 g}}}
$$

L'équation 9 est l'équation de travail du pycnomètre à gaz, elle permet de connaître le volume $\mathrm{V}$ occupé par l'échantillon, le volume de la cellule $\mathrm{V}_{\text {cell }}$ et le volume additionel $\mathrm{V}_{\text {add }}$ étant déterminés par etalonnage (à $0,02 \%$ près et contrôlés régulièrement).

Le pycnomètre à gaz utilisé est un Micromeritics A1330, fonctionnant de manière automatique. Avant chaque série de mesure du volume V (une série représente 15 mesures individuelles de V), un cycle de 10 purges par remplissage de la cellule de mesure à l'hélium à température ambiante est effectué.

\section{$\sin 2$}

\section{Application aux matériaux argileux}

Expérimentalement, ce volume $\mathrm{V}$ correspond à tout le volume non rempli par l'air, c'est-à-dire le volume

$$
\mathrm{V}=\mathrm{V}_{\mathrm{s}}+\mathrm{V}_{\mathrm{w}}=\mathrm{V}_{\text {totat }}-\mathrm{V}_{\text {air }}
$$

On ne peut donc mesurer directement $V_{s}$ et donc en déduire la masse volumique de solide $\rho_{s^{\prime}}$ définie par :

$$
\rho_{s}=\frac{M_{s}}{V_{s}}
$$

Soit $M_{h}$ la masse mise en jeu, il s'agit de la masse humide : $M_{h}=M_{S}+M_{w}$. On mesure le volume $\mathrm{V}$ occupé $\operatorname{par} M_{h}$; la masse volumique expérimentale $\rho$ est donc :

$$
\rho=\frac{M_{w}+M_{s}}{V_{w}+V_{s}}
$$

Compte tenu des définitions classiques de la masse volumique de l'eau et de la teneur en eau :

$\rho_{w}=\frac{M_{w}}{V_{w}}$ et $w=\frac{M_{w}}{M_{s}} ;$ l'équation 12 s'écrit :

$$
\rho=\frac{M_{w}+M_{s}}{\frac{M_{w}}{\rho_{w}}+\frac{M_{s}}{\rho_{s}}}
$$

D'où finalement :

$$
\rho=\frac{1+w}{\frac{w}{\rho_{w}}+\frac{1}{\rho_{s}}}
$$

On en déduit la masse volumique de solide $\rho_{s^{\prime}}$ valeur que l'on cherche à déterminer :

$$
\rho_{s}=\frac{\rho}{1+w \cdot\left(1-\frac{\rho}{\rho_{w}}\right)}
$$

II en résulte qu'il est possible a priori de déterminer la masse volumique solide pour n'importe quelle valeur de la teneur en eau : connaissant la teneur en eau et la masse volumique de l'eau, on devrait aboutir à une masse volumique de solide invariante.

Expérimentalement, on constate que ce n'est pas le cas : pour l'échantillon GREC pris comme exemple, et en prenant une masse volumique de l'eau égale à $1,00 \mathrm{~g} / \mathrm{cm}^{3}$, la masse volumique solide augmente simultanément à la teneur en eau au lieu de rester constante (Tableau III).

L'écart constaté est trop important pour pouvoir être imputé à des erreurs de mesure des pressions, de la masse d'échantillon ou de la teneur en eau. Tous les autres échantillons étudiés montrent un comportement identique, en utilisant une masse volumique de l'eau égale à $1,00 \mathrm{~g} / \mathrm{cm}^{3}$.

Afin de déterminer plus aisément l'origine de cet écart, on opère un changement de variable on utilise la masse volumique sèche de solide $\rho_{d^{\prime}}$ selon sa définition usuelle (on rappelle que, dans le cas des mesures au pycnomètre à hélium, le volume d'air est nul) :

$$
\rho_{d}=\frac{\rho}{1+W}=\frac{m_{s}}{V_{s}+V_{w}}
$$

ou, plus précisément, le volume spécifique sec $\Omega_{d^{\prime}}$ qui est l'inverse de la masse volumique sèche :

$$
\Omega_{d}=\frac{1}{\rho_{d}}=\frac{V_{s}+V_{w}}{m_{s}}
$$


TABLEAU III Résultats expérimentaux sur l'échantillon GREC premiers calculs pour déterminer $\rho_{s}$ en prenant $\rho_{w}=1,00 \mathrm{~g} / \mathrm{cm}^{3}$

\begin{tabular}{cccc} 
\% HR & $w(\%)$ & $\rho\left(\mathrm{g} / \mathrm{cm}^{3}\right)$ & $\begin{array}{c}\rho\left(\mathrm{g} / \mathrm{cm}^{3}\right) \\
\left.\text { (éq. } 15 \text { avec } \rho_{\mathrm{w}}=1,00 \mathrm{~g} / \mathrm{cm}^{3}\right)\end{array}$ \\
\hline 7 & 4,71 & 2,3886 & 2,5557 \\
\hline 12 & 5,82 & 2,3659 & 2,5703 \\
\hline 23 & 7,87 & 2,3239 & 2,5942 \\
\hline 33 & 8,85 & 2,3101 & 2,6132 \\
\hline 44 & 10,15 & 2,2897 & 2,6347 \\
\hline 54 & 11,67 & 2,2730 & 2,6695 \\
\hline 75 & 15,86 & 2,1876 & 2,6953 \\
\hline 90 & 22,16 & 2,0779 & 2,7299 \\
\hline 97 & 28,76 & 1,9872 & 2,7751
\end{tabular}

Le volume spécifique sec présente donc tout son intérêt, car il traduit directement le volume occupé par l'assemblage eau + solide pour une masse déterminée de solide. Quand la teneur en eau change, il n'y a pas migration des éléments constitutifs du feuillet (donc de la phase solide) vers l'extérieur (la phase liquide) : la masse de la phase solide est invariante. Le volume spécifique sec constitue donc une meilleure base de référence pour tous les calculs, de préférence à la masse volumique sèche.

Le volume spécifique sec s'écrit donc :

$$
\Omega_{d}=\frac{1+W}{\rho}
$$

D'une manière similaire, on définit les volumes spécifiques de l'eau $\Omega_{\mathrm{w}}$ et du solide $\Omega_{\mathrm{s}}$ comme les inverses des masses volumiques respectives :

$$
\begin{aligned}
& \Omega_{w}=\frac{1}{\rho_{w}}=\frac{V_{w}}{m_{w}} \\
& \Omega_{s}=\frac{1}{\rho_{s}}=\frac{V_{s}}{m_{s}}
\end{aligned}
$$

En utilisant ces définitions, l'équation 14 se transforme en :

$$
\rho=\frac{1+W}{W \cdot \Omega_{w}+\Omega_{s}}
$$

Avec la définition du volume spécifique sec (éq. 18), on a donc finalement :

$$
\frac{1}{\Omega_{d}}=\frac{1}{W \cdot \Omega_{w}+\Omega_{s}}
$$

d'où :

$$
\Omega_{d}=W \cdot \Omega_{w}+\Omega_{s}
$$

On a donc une fonction linéaire reliant la masse volumique expérimentale $\rho$ à la teneur en eau w et à la masse volumique de solide $\rho_{s}$. Les paramètres de cette droite, calculés par régression linéaire, s'en déduisent aisément : l'ordonnée à l'origine $(w=0)$ est égale à l'inverse de la masse volumique de solide (éq. $23: \Omega_{d}=$ $\Omega$ ) et la pente est égale à l'inverse de la masse volumique de l'eau (éq. 19).
Inversement, l'équation 23 peut s'exprimer sous une forme plus classique en mécanique des sols :

$$
\rho_{d}=\frac{1}{w \cdot \Omega_{w}+\Omega_{s}}=\frac{1}{\frac{W}{\rho_{w}}+\frac{1}{\rho_{s}}}
$$

En traçant la courbe $\rho_{d}=\mathrm{f}(w)$, on retrouve la représentation classiquement utilisée lors des essais Proctor, avec le tracé de l'hyperbole des isovaleurs du taux de saturation (et notamment pour $\mathrm{Sr}=1$, car on n'a pas de volume occupé par l'air). Cette courbe coïncide avec l'axe des ordonnées pour une valeur de la teneur en eau nulle pour donner également la valeur de la masse volumique de solide.

\section{5}

\section{Résultats}

Chaque matériau est mis en équilibre à différentes humidités relatives contrôlées (dessiccateurs avec des solutions salines saturées) sur une gamme s'étendant de 7 à $97 \%$ HR et maintenus à une température de $22 \pm$ $2{ }^{\circ} \mathrm{C}$. Les différents sels utilisés sont : $\mathrm{NaOH}(7 \%), \mathrm{LiCl}$ $(12 \%), \mathrm{CH}_{3} \mathrm{COOK}(23 \%), \mathrm{MgCl}_{2}(33 \%), \mathrm{K}_{2} \mathrm{CO}_{3}(44 \%)$, $\mathrm{Mg}\left(\mathrm{NO}_{3}\right)_{2}(54 \%), \mathrm{NaCl}(75 \%), \mathrm{BaCl}_{2}(90 \%)$ et $\mathrm{K}_{2} \mathrm{SO}_{4}$ $(97 \%)$. Après plusieurs semaines de mise en équilibre, les poudres sont directement introduites dans le pycnomètre à hélium, et une série de 15 mesures successives du volume $\mathrm{V}$ est effectuée, à partir desquelles la masse volumique moyenne $\rho$ est calculée. Dès que la mesure au pycnomètre est terminée, un contrôle de la prise d'essai est effectué, afin de vérifier qu'il n'y a pas eu de variation de la masse, qui se sont toutes révélées négligeables (au maximum 0,1\%). Cette prise d'essai est ensuite directement mise à $105^{\circ} \mathrm{C}$ pour une détermination classique de la teneur en eau.

Les isothermes de sorption d'eau (Fig. 3) montrent le comportement type des smectites. Les smectites calciques (GREC, TURK et PROV) présentent une sorption d'eau plus importante que celles sodiques (WYO1 et WYO2) dans le domaine des faibles humidités relatives, confirmant les études antérieures (Chiou et Rutherford, 1997 ; Jouany, 1981 ; Touret, 1988 ; Turki, 1983). Pour les plus fortes humidités relatives, la différence est moindre ; l'argile turque restant néanmoins largement plus hydratée en raison de sa pureté en smectite.

Les courbes caractéristiques des masses volumiques et des volumes spécifiques secs des cinq matériaux étudiés montrent une bonne corrélation avec les expressions correspondantes (les coefficients de régression sont supérieurs à 0,99 ). Dans tous les cas, on peut déterminer aisément la masse volumique de solide. Celle-ci varie entre 2,529 et $2,669 \mathrm{~g} / \mathrm{cm}^{3}$. Pour des smectites pures, les masses volumiques de solide sont généralement de l'ordre de $2,7 \mathrm{~g} / \mathrm{cm}^{3}$ avec des valeurs plus élevées dans le cas des smectites riches en fer (Tessier, 1984). Les masses volumiques plus faibles obtenues sur les cinq matériaux étudiés s'expliquent, outre la composition chimique propre à chaque smectite, par la présence des phases minérales non argileuses : feldspaths, gypse, etc. Des essais de masse volumique effectués sur la poudre séchée à $105^{\circ} \mathrm{C}$ et refroidie en atmosphère anhydre confirment les valeurs obtenues par extrapolation à une teneur en eau nulle : l'erreur maximale n'excède pas $0,01 \mathrm{~g} / \mathrm{cm}^{3}$. 


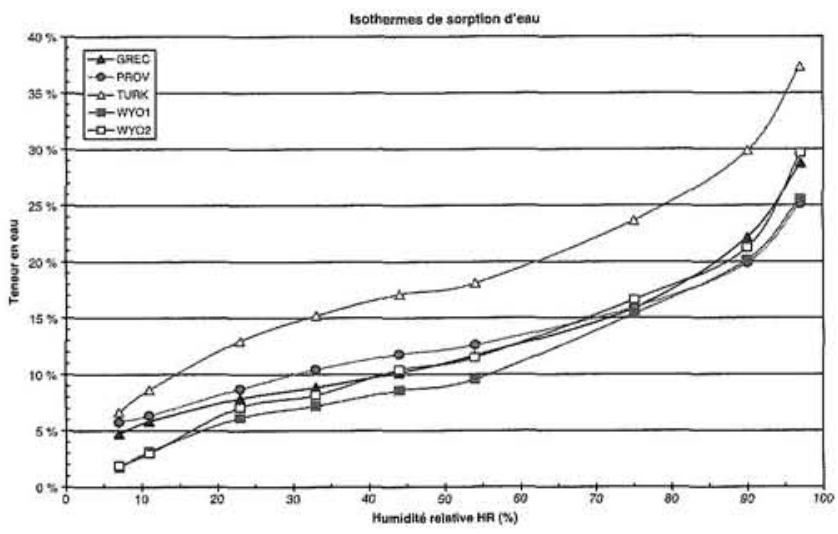

FIG. 3 Isothermes de sorption d'eau des cinq smectites.

Water sorption of the five smectites studied.

Les valeurs de la pente des droites de régression linéaire constituent également un point marquant de ces courbes. En effet, la pente correspond au volume spécifique de l'eau $\Omega_{\mathrm{w}}$ (éq. 23), dont le calcul donne une valeur systématiquement inférieure à celle de l'eau pure. A la température de l'essai $\left(27 \pm 1{ }^{\circ} \mathrm{C}\right)$, la masse volumique de l'eau pure est de $0,9965 \pm 0,0003 \mathrm{~g} / \mathrm{cm}^{3}$, soit un volume spécifique de $\Omega_{\mathrm{w}} 1,0035 \mathrm{~cm}^{3} / \mathrm{g}$. La pente de la droite calculée par régression devrait correspondre à cette valeur (éq. 23). Or, ce n'est pas le cas : dans l'exemple de la courbe de l'échantillon WYO2 (Fig. 4), le volume spécifique de l'eau pour cet échantillon est déterminé égal à $0,8795 \mathrm{~cm}^{3} / \mathrm{g}$, soit une masse volumique de l'eau de $1,14 \mathrm{~g} / \mathrm{cm}^{3}$. Tous les autres échantillons montrent un comportement identique. Dans l'ensemble, le volume spécifique de l'eau est compris entre 0,84 et $0,89 \mathrm{~cm}^{3} / \mathrm{g}$, soit des valeurs de 15 à $20 \%$ inférieures à celles de l'eau pure. Ceci correspond à des masses volumiques de l'eau comprises entre 1,1 et $1,2 \mathrm{~g} / \mathrm{cm}^{3}$ (Tableau IV).

Pour interpréter ceci, l'évolution de la position de la raie 001 des smectites a été mesurée simultanément à la mesure de la masse volumique. Pour l'état déshydraté $(0 \% \mathrm{HR})$, la smectite présente une distance basale $\mathrm{d}_{001}$ de l'ordre de $10 \AA$; ceci correspond à l'état de référence ( $\mathrm{sec}$ ) et donc, par définition, à la masse volumique de grains. Cette distance est la somme de l'épaisseur du

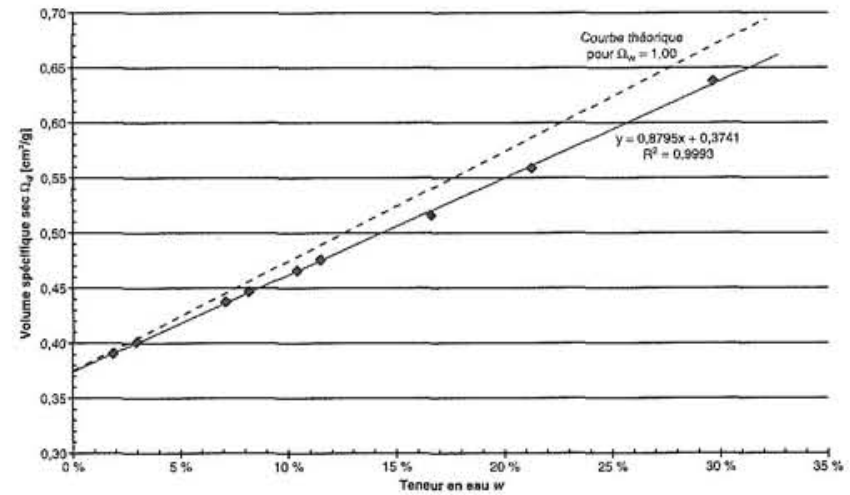

FIG. 4 Évolution du volume spécifique sec de l'échantillon WYO2 en fonction de la teneur en eau losanges : points expérimentaux et droite de régression linéaire correspondante), droite discontinue: courbe théorique de même ordonnée à l'origine $\Omega s=0,3741$ mais avec une pente $\Omega_{\mathrm{w}}=1,00$.

Evolution of the dry specific volume (sample WYO2) versus water content (diamond: experimental points and its full line: linear regression curve), dashed line: theorical curve with $\Omega_{\mathrm{s}}=0.3741$ and $\Omega_{\mathrm{w}}=1.00$.

feuillet et de l'épaisseur de l'espace interfoliaire (ne contenant pas d'eau pour $0 \% \mathrm{HR}$ ). Quand l'humidité relative augmente jusqu'à environ $50-70 \% \mathrm{HR}$, la distance $\mathrm{d}_{o \text { op }}$ passe à $12,6 \AA$ dans le cas d'une smectite sodique (ou à $15 \AA$ dans le cas d'une smectite calcique): on est donc en présence d'un gonflement (qui va également être visualisé à l'échelle macroscopique). En corrélant ceci à l'évolution de la teneur en eau, on démontre aisément que l'eau mise en jeu est bien l'eau qui a pénétré dans l'espace interfoliaire. Ici, l'eau interparticulaire ne joue qu'un rôle secondaire dans le domaine des humidités relatives considérées. La pente des droites correspond donc au volume spécifique de l'eau interfoliaire, qui est plus faible que celui de l'eau libre.

Les mesures montrent également que la masse volumique de l'eau interfoliaire dépend de la nature du cation échangeable, et plus particulièrement de la répartition des ions, exprimée par le rapport $\mathrm{Na}$ échan-

TABLEAUIV Résultats expérimentaux : teneurs en eau $w$ (en \%) et masses volumiques $\rho$ mesurées au pycnomètre à hélium $\left(\mathrm{en} \mathrm{g} / \mathrm{cm}^{3}\right)$ pour les différentes humidités relatives; et calcul des coefficients des courbes de régression de l'équation 24.

\begin{tabular}{|c|c|c|c|c|c|c|c|c|c|c|}
\hline & \multicolumn{2}{|c|}{ GREC } & \multicolumn{2}{|c|}{ PROV } & \multicolumn{2}{|c|}{ TURK } & \multicolumn{2}{|c|}{ WYO1 } & \multicolumn{2}{|c|}{ WYO2 } \\
\hline & W & $p$ & W & $\rho$ & W & $p$ & W & $\rho$ & w & $\rho$ \\
\hline $7 \%$ HR & 4,71 & 2,3886 & 5,75 & 2,4506 & 6,65 & 2,3926 & 1,74 & 2,592 & 1,89 & 2,6080 \\
\hline $12 \% \mathrm{HR}$ & 5,82 & 2,3659 & 6,33 & 2,4326 & 8,63 & 2,3337 & 3,13 & 2,5583 & 2,97 & 2,5682 \\
\hline $23 \% \mathrm{HR}$ & 7,87 & 2,3239 & 8,70 & 2,3749 & 12,97 & 2,2483 & 6,14 & 2,4718 & 7,08 & 2,4477 \\
\hline $33 \% \mathrm{HR}$ & 8,85 & 2,3101 & 10,44 & 2,3427 & 15,21 & 2,2175 & 7,21 & 2,4475 & 8,16 & 2,4224 \\
\hline $44 \% \mathrm{HR}$ & 10,15 & 2,2897 & 11,77 & 2,3147 & 17,09 & 2,1915 & 8,57 & 2,4150 & 10,40 & 2,3699 \\
\hline $54 \% \mathrm{HR}$ & 11,67 & 2,2730 & 12,60 & 2,3007 & 18,09 & 2,1741 & 9,55 & 2,3930 & 11,48 & 2,3434 \\
\hline $90 \% \mathrm{HR}$ & 22,16 & 2,0779 & 19,87 & 2,1730 & 29,86 & 2,0247 & 20,16 & 2,1669 & 21,25 & 2,1704 \\
\hline $97 \%$ HR & 28,76 & 1,9872 & 25,13 & 2,0974 & 37,31 & 1,9319 & 25,55 & 2,0869 & 29,66 & 2,0321 \\
\hline $\begin{array}{l}r^{2} \\
r_{s} \\
r_{w}\end{array}$ & \multicolumn{2}{|c|}{$\begin{array}{c}0,9921 \\
2,529 \mathrm{~g} / \mathrm{cm}^{3} \\
1,160 \mathrm{~g} / \mathrm{cm}^{3}\end{array}$} & \multicolumn{2}{|c|}{$\begin{array}{c}0,9996 \\
2,610 \mathrm{~g} / \mathrm{cm}^{3} \\
1,181 \mathrm{~g} / \mathrm{cm}^{3}\end{array}$} & \multicolumn{2}{|c|}{$\begin{array}{c}0,9977 \\
2,561 \mathrm{~g} / \mathrm{cm}^{3} \\
1,178 \mathrm{~g} / \mathrm{cm}^{3}\end{array}$} & \multicolumn{2}{|c|}{$\begin{array}{c}0,9977 \\
2,662 \mathrm{~g} / \mathrm{cm}^{3} \\
1,142 \mathrm{~g} / \mathrm{cm}^{3}\end{array}$} & \multicolumn{2}{|c|}{$\begin{array}{c}0,9980 \\
2,669 \mathrm{~g} / \mathrm{cm}^{3} \\
1,143 \mathrm{~g} / \mathrm{cm}^{3}\end{array}$} \\
\hline
\end{tabular}




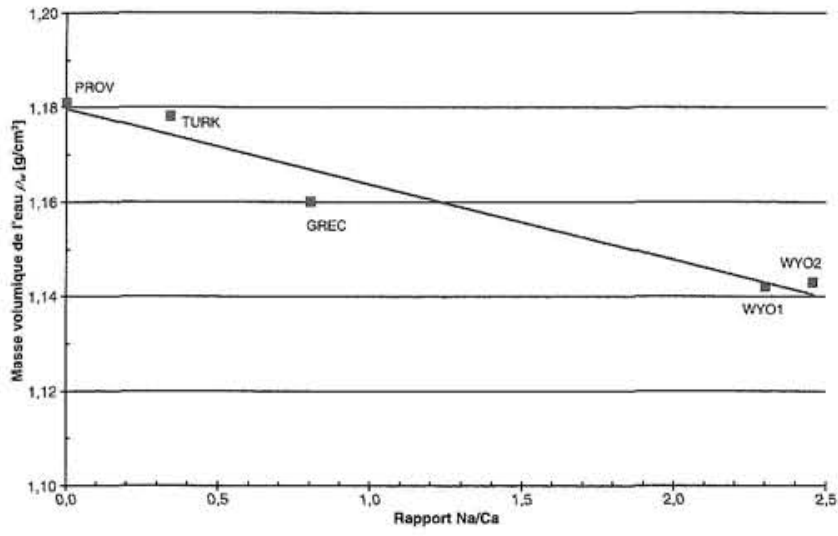

FIG.5 Influence du rapport $\mathrm{Na} / \mathrm{Ca}$ sur la masse volumique de l'eau mesurée.

Influence of $\mathrm{Na} / \mathrm{Ca}$ ratio on the measured water density.

geable/Ca échangeable (Tableau II et Fig. 5). Elle est plus élevée pour les smectites calciques que pour les smectites sodiques.

\section{5 \\ Conclusion}

Par mesure de la masse volumique de l'eau et de l'argile, nous démontrons expérimentalement que l'eau présente dans l'espace interfoliaire ne peut donc pas être assimilée à de l'eau libre. Cette conclusion rejoint celle de Karaboni et al. (1996) qui couplent leurs simulations de Monte-Carlo et de dynamique moléculaire à l'évolution de la distance $\mathrm{d}_{001}$ pour une montmorillonite sodique (pour des distances $\mathrm{d}_{001}$ inférieures à $25 \AA$, et donc dans les mêmes conditions que la présente étude).

A l'échelle macroscopique, cette eau interfoliaire présente une masse volumique plus élevée que celle de l'eau libre, avec des valeurs plus fortes pour les smectites calciques et/ou magnésiennes que pour les smectites sodiques. Skipper et al. (1991) trouvent la même tendance : leurs simulations numériques donnent des valeurs de masse volumique de $1,38 \pm 0,04 \mathrm{~g} / \mathrm{cm}^{3}$ pour une smectite magnésienne et $1,14 \pm 0,04 \mathrm{~g} / \mathrm{cm}^{3}$ pour une smectite sodique.

Les résultats obtenus dans cette étude confirment que l'eau interfoliaire présente une organisation particulière autour des cations échangeables situés dans l'espace interfoliaire. Ces études antérieures utilisent des méthodes aussi diverses que l'infrarouge (Prost, 1975 ; Yan et al., 1996), les simulations numériques (Skipper et al., 1995a ; Skipper et al., 1995b), la DRX seule (Kraehenbuehl et al., 1987 ; Moore et Reynolds, 1997) ou coupée à la diffusion des rayons $X$ aux petits angles (Pons, 1980).

Par ailleurs, l'absence de rupture de pente sur le domaine de HR exploré montre qu'il n'est pas possible de distinguer deux types d'eau : d'une part, l'eau « liée » située dans l'espace interfoliaire et, d'autre part, l'eau ( libre ) avec des propriétés (telles que la masse volumique) identiques à l'eau pure. Dans l'ensemble de l'espace interfoliaire des smectites, il existe donc des
( îlots ) (clusters) hydratés, dont le nombre augmente au fur et à mesure que l'humidité relative augmente (la distance $\mathrm{d}_{001}$ reste constante), jusqu'à ce que tout l'espace interfoliaire soit rempli par un film continu d'eau. Cette représentation, appliquée historiquement en mécanique des sols pour expliquer les phénomènes capillaires entre les particules argileuses, s'étend ici au niveau interfoliaire et, donc à une plus petite échelle (l'espace interfoliaire n'est large que de quelques angströms au plus). Mais ceci n'implique pas que les lois de capillarité puissent s'appliquer dans l'espace interfoliaire où les interactions physico-chimiques jouent un rôle beaucoup plus significatif.

Pour les propriétés relevant de l'aspect hydro-mécanique des argiles, les principales conséquences pratiques d'une masse volumique de l'eau plus élevée que l'eau « normale ») sont :

- pour une même teneur en eau et une même masse volumique sèche, le degré de saturation, fonction inverse de la masse volumique de l'eau, sera plus faible (d'environ 10-15\%). Par exemple, une éprouvette d'argile GREC de masse volumique sèche $1,70 \mathrm{~g} / \mathrm{cm}^{3}$ et de teneur en eau de $5 \%$ présente un taux de saturation classique (avec $\rho_{w}=1,00 \mathrm{~g} / \mathrm{cm}^{3}$ ) égal à 0,78 . En prenant en compte la masse volumique déterminée pour cette argile $\left(\rho_{w^{\prime}}=1,16 \mathrm{~g} / \mathrm{cm}^{3}\right)$, le calcul donne un taux de saturation égal à 0,67 . Ceci correspond à un remplissage de la porosité (par l'eau) moindre que prévu, alors que la masse d'eau reste constante. Dans le cas d'une migration chimique se faisant essentiellement dans le volume de la phase ( eau »), la vitesse de transport des éléments serait donc plus faible ;

- dans le domaine des humidités relatives jusqu'à $97 \%$ au moins, la pression capillaire, exprimée en MPa sera plus élevée en valeur absolue (+ 15 à $+20 \%)$, alors que le potentiel osmotique (exprimé en $\mathrm{m}$ ) ne sera pas affecté ;

- comme corollaire des deux propriétés précédentes, la courbe capillaire reliant le degré de saturation $\mathrm{Sr}$ à la pression capillaire sera translatée ;

- la principale difficulté à résoudre sur les courbes capillaires $\mathrm{Sr}=\mathrm{f}$ (P capillaire) sera de raccorder le domaine des pressions capillaires correspondant aux humidités relatives de 5 à $97 \%$ (i.e. de 400 à environ $4 \mathrm{MPa}$ ) aux pressions capillaires plus faibles (supérieures à $97 \% \mathrm{HR}$ ), pour lesquelles l'eau mise en jeu est l'eau ( libre ) avec ses caractéristiques standard. Cette transition entre les deux types d'eau serait mise en évidence par une rupture de la pente de la courbe $\Omega_{d}=f(w)$. A partir d'une certaine valeur de $W$, l'évolution de $\Omega_{d}$ suivrait la valeur du volume spécifique de l'eau pure. Ce passage de l'état non saturé à l'état saturé reste à appréhender.

La détermination simultanée de la masse volumique de l'eau et du solide, telle que présentée lors de cette étude, a permis d'obtenir deux paramètres physiques qui sont réellement représentatifs du matériau mis en œuvre, car on a vu que la masse volumique de l'eau variait en fonction de la composition des ions de l'espace interfoliaire. 
Ben Rhaiem H., Tessier D., Pons C.H. Comportement hydrique et évolution structurale des montmorillonites lors d'un cycle de dessiccation-humectation partie I: Cas des montmorillonites cal ciques. Clay Minerals 21, 1986, p. 9-29.

Berend I. - Les mécanismes d'hydratation de montmorillonites homoioniques pour des pressions relatives inférieures à 0,95 . Thèse, Institut national polytechnique de Lorraine, Nancy, 1991, 332 p.

Chantret F., Desprairies A., Douillet P. Jacob C., Sberg M., Trauth N. - Révision critique de l'utilisation des méthodes thermiques en sédimentologie : cas des smectites (montmorillonites). Bulletin du Groupe français des argiles XXIII, 1971, p. 14-2.

Chiou C.T., Rutherford D.W. - Effect of exchanged cation and layer charge on the sorption of water and EGME vapors on montmorillonite clay. Clays and Clay minerals 45, 1997, p. 86-880.

Glaeser R., Mering J. - Domaines d'hydratation homogènes des smectites. Compte rendu de l'Académie des sciences 267, Paris, 1968, p. 463-466.

Jouany C. - Transferts d'eau par évaporation dans les milieux argileux. Thèse, université Paul-Sabatier, Toulouse, 1981 $102 \mathrm{p}$.

Karaboni S.B.S., Heidug W., Uy J., Van Oort E. - The swelling of clay: molecular simulations of the hydration of montmorillonite. Science 271,1996 , p. 1102 1104.
Kraehenbuehl F., Stoeckli H.F., Brunner F, Kahr G., Müller-Von Moss M. - Study of the water-bentonite system by vapour adsorption, immersion calorimetry and X-ray techniques - I: Micropore volumes and internal surface areas, following Dubinin's theory. Clay Minerals 22, 1987, p. 1-9.

Moore D.M., Reynolds R.C. - X-ray diffraction and the identification and analysis of clay minerals. Oxford University Press, Oxford, 1997, 380 p., 2nd ed.

Pons C.-H. - Mise en évidence des relations entre la texture et la structure dans les systèmes eau-smectites par diffusion aux petits angles du rayonnement $X$ synchroton. Thèse, université d'Orléans, 1980, $175 \mathrm{p}$.

Prost R. - Etude de l'hydratation des argiles: interactions eau-minéral et mécanisme de la rétention de l'eau. Thèse, université Paris VI, 1975, 135 p.

Raynal J. - Recherche de méthodes d'analyse thermique pour le dosage des minéraux associés aux argiles. Mémoire d'ingénieur du Conservatoire national des arts et métiers, Paris, 1989, 149 p.

Robinet J.-C., Pasquiou A., Jullien A., Belanteur N., Plas F. - Expériences de laboratoire sur le comportement thermo-hydro-mécanique de matériaux argileux remaniés gonflants et non gonflants. Revue française de géotechnique 81, 1997, p. 53-80.

Skipper N.T., Refson K., McConnel J.D.C. - Computer simulation of interlayer water in 2:1 clay. Journal of Chemical Physics 94, 1991, p. 434-445.

Skipper N.T., Chang F.-R.C., Sposito G. Monte-Carlo simulations of interlayer molecular structure in swelling clay minerals : 2. Monolayer hydrates. Clays and Clay Minerals 43, 1995(a), p. 294-303. Skipper N.T., Sposito G., Chang F.-R.C. - Monte-Carlo simulations of interlayer molecular structure in swelling clay minerals ; 1 . Methodology. Clays and Clay Minerals 43, 1995(b), p. 285293.

Tessier D. - Étude expérimentale de I'organisation des matériaux argileux ; hydratation, gonflement et structuration au cours de la dessiccation et de la réhumectation. Thèse, université Paris VII, 1984, $361 \mathrm{p}$.

Touret O. - Structure des argiles hydratées: thermodynamique de la déshydratation et de la compaction des smectites. Thèse, université Louis-Pasteur, Strasbourg, 1988, $170 \mathrm{p}$.

Trauth N. - Contribution à l'étude des montmorillonites à haute température. Thèse, faculté des sciences, Strasbourg, $1964,82 \mathrm{p}$

Turki F. - Gonflement et cinétique d'imbibiton de quelques argiles. Thèse, université Louis-Pasteur, Strasbourg, 1983, $103 \mathrm{p}$.

Yan L., Roth C.B., Low P.F. - Changes in the $\mathrm{Si}-\mathrm{O}$ vibrations of smectites layers accompanying the sorption of interlayer water. Langmuir 12, 1996, p. 4442-4429. 